\title{
ANÁLISE DOS ARTIGOS PUBLICADOS EM EVENTOS CONTÁBEIS QUALIFICADOS PELA CAPES COMO "E1" SOBRE AVALIAÇÃO DE DESEMPENHO NO SETOR PÚBLICO
}

\author{
ANALYSIS OF ARTICLES PUBLISHED AT ACCOUNTING EVENTS QUALIFIED BY CAPES AS \\ "E1" ABOUT TO THE PERFORMANCE EVALUATION IN THE PUBLIC SECTOR
}

Almir Rodrigues Durigon

almir durigon@hotmail.com

Universidade do Estado do Mato Grosso
Carlos Alberto Diehl

$\underline{\text { cd@unisinos.br }}$

Universidade do Vale do Rio dos Sinos

\section{RESUMO}

Este artigo tem por objetivo analisar as características das publicações referentes à avaliação de desempenho no setor público, publicadas nos eventos da área contábil qualificadas pela Capes com conceito "E1" (Congresso USP de Controladoria e Contabilidade - CCC-USP, Congresso Brasileiro de Custos - CBC, Encontro da Associação Nacional de Pós - Graduação e Pesquisa em Administração - ENANPAD, e Associação Nacional dos Programas de Pós-Graduação em Ciências Contábeis ANPCONT). A metodologia adotada constitui-se de pesquisa bibliográfica e de análise de conteúdo. Foram analisados os artigos publicados nas edições de 2004 a 2011 do CCC-USP, as edições de 2002 a 2011 do ENANPAD, as edições de 2001 a 2010 do CBC, e as edições de 2007 a 2011 da ANPCONT. Os resultados da pesquisa demonstram que a caracterização da natureza e utilidade da avaliação de desempenho no setor público como uma prática que viabilize maior eficiência e eficácia na execução das atividades de apoio é um tema pouco presente nos artigos dos congressos analisados. Outra importante constatação é a de que os artigos analisados utilizam como metodologia básica para o desenvolvimento dos trabalhos o estudo de caso e a pesquisa bibliográfica. O estudo conclui que as publicações encontradas são consideradas um número pequeno e não apresentam relevância para uma análise crítica do tema de modo que oferece hipóteses mais significativas para a evolução e aperfeiçoamento dessa prática de gestão.

Palavras - Chaves: Avaliação de desempenho. Setor Público. Publicação. Artigos. Pesquisa.

\section{ABSTRACT}

This article aims at analyzing the characteristics of publications referring to the performance evaluation in the publicsector, published at accounting events qualified by Capes as concept "E1" (Congress USP of Controller and Accounting - CCC - USP, Brazilian Congress of Costs - CBC, Meeting of National Association of Post-Graduation and Research in Administration - ENANPAD, and National Association of Post-Graduation Programs in Accounting - ANPCONT). The adopted methodology is constituted by bibliographic research as well as content analysis. We have analyzed articles published in the editions from 2004 to 2011 of CCC-USP, editions from 2002 to 2011 of ENANPAD, editions from 2001 to 2010 of CBC, and editions from 2007 to 2011 of ANPCONT. The results of the research have shown that the characterization of nature and utility of performance evaluation in the public sectoras a practice that allows greater efficiency in the execution of support activities is a theme not so present in the articles of the analyzed congresses. Another important observation regards to the fact that the analyzed articles utilize, as basic methodology, the case study and bibliographic research. The study concludes that the publications found are considered a small figure, which does not show relevance for a critical analysis of the theme so that it offers more significant hypothesis for the evolution and improvement of this management practice.

Keywords: Performance Evaluation. Public Sector. Publication. Articles. Research. 
Artigo recebido em: 10.04.2014; Aceito em: 07.07.2014

\section{INTRODUÇÃO}

A criação de mecanismos para avaliar o desempenho nas organizações não é recente. Reforçando esse entendimento, Figueiredo et al. (2005) aduzem que o sistema de avaliação de desempenho passou por três fases distintas: a primeira fase foi do século XIV ao século XIX, quando se originaram as primeiras práticas de Contabilidade com a finalidade de controlar os processos produtivos. A segunda fase se deu por volta do início do século XX, se estendendo até meados da década de 1980, quando o processo de avaliação de desempenho integrou formalmente o ciclo de planejamento e controle. E a terceira teve início durante a década de 1980, permanecendo até os dias atuais, quando diversas críticas foram feitas ao modelo tradicional e novos modelos foram propostos com várias dimensões.

Já Para Ghalayini e Nobles (1996) a avaliação de desempenho teve duas fases principais. A primeira fase começou no final dos anos de 1880 e atravessou a década de 1980. Nessa fase a ênfase era sobre as medidas financeiras como lucro, retorno sobre investimento e produtividade. A segunda fase começou na década de 1980, como um resultado de alterações no mercado mundial. As empresas começaram a perder parcela de mercado para concorrentes estrangeiros que foram capazes de fornecer produtos de qualidade superior, com custos mais baixos e com maior variedade.

Tezza, Bornia e Vey (2010) acreditam que a Contabilidade era usada como sistema de avaliação de desempenho e que esse sistema era usado antes dos paradigmas fordista-taylorista e, antes mesmo da revolução industrial, pelos artesãos. Para os autores, no início do século XX, os gerentes de fábricas tomavam decisões com base nas informações sobre o custo por hora de transformação de matériaprima em produtos acabados. Com isso criava-se a possibilidade em medir a eficiência do processo e avaliava-se o desempenho de cada funcionário. Baseada nessas informações era concedida a remuneração adicional aos mais produtivos.

No século atual há um grande interesse por parte dos gestores em investigar sistemas de avaliação de desempenho, no intuito de fortalecer e deixar a organização mais competitiva. Nesse contexto, de conhecer sistemas de avaliação, Lima e Ponte (2006) afirmam que nos dias atuais, há uma grande procura por conhecimentos relacionados à avaliação de desempenho e que instrumentos a ele relacionados vêm sendo pesquisados pelos gestores de organizações públicas e privadas, preocupados em avaliar o desempenho destas organizações.

É fato que transformações substanciais foram realizadas nas políticas de gestão pública e as reformas administrativas propostas trazem novos discursos e aprendizados originados no setor privado cujo objetivo é direcionar a organização na busca por melhores resultados e desempenhos (SECCHI, 2009). Na busca por estes, Martins et al. (2010) explicam que no âmbito do setor público brasileiro foram aprovadas legislações acerca de avaliação de desempenho desde a década de 1930, no entanto, foi a partir de 1998, com a Emenda Constitucional 19, que ocorreu a inserção em nível constitucional. Porém, ainda há pouca pesquisa neste campo, o que dificulta um maior avanço da avaliação de desempenho no setor público (LYRIO; ENSSLIN; DUTRA, 2007).

Para Diehl e Souza (2008), os eventos científicos (congressos, palestras, seminários, painéis, encontros entre outros) são ocasiões oportunas para os autores expressarem suas opiniões, e através dos conceitos que se contradizem e se complementam, contribuírem para o crescimento do conhecimento científico.

Considerando a importância dos eventos científicos qualificados pela Capes como "E1", julga-se relevante questionar: quais as características das publicações sobre avaliação de desempenho no setor público? Para respondê-la este artigo objetiva analisar as características das publicações referentes à 
avaliação de desempenho no setor público, publicadas nos eventos da área contábil, qualificadas pela Capes como "E1".

Tal conhecimento permiti investigar os avanços teóricos e práticos sobre o tema. É uma forma também de dar visibilidade ao desenvolvimento científico produzido no Brasil, na área contábil. A justificativa de estudos como esse se faz pela importância que há na busca pela eficiência dos processos e pela eficácia dos resultados da gestão pública, com vistas a otimizar o uso dos recursos postos à disposição dos gestores.

Além disso, este estudo surge em complemento a diversos outros semelhantes na literatura de gestão, destacando-se Keinert (2000), Pacheco (2003), Lyrio, Ensslin e Dutra (2007), Hocayen-da-silva, Rossoni e Ferreira Junior (2008), e Martins et al. (2010).

Este artigo encontra-se estruturado em mais quatro seções. Na segunda seção apresenta-se o referencial teórico sobre avaliação de desempenho. Posteriormente, classifica-se a metodologia aplicada a fim de se atingir os desígnios da pesquisa. A quarta seção traz a apresentação e analise dos resultados e, por último, as considerações finais e referências.

\section{REVISÃO DA LITERATURA}

\subsection{Avaliação de desempenho}

Avaliação de desempenho é um conceito multidisciplinar, trabalhado por várias áreas do conhecimento, tais como Engenharia, Administração, Ciências Contábeis e outras (KIYAN, 2001). Já para Carpinetti (2000) avaliação de desempenho trata de métodos para quantificar a eficiência e a eficácia das atividades de um negócio por meio de métricas ou indicadores de desempenho. Neely, Gregory e Platts (1995) definem avaliação de desempenho como procedimento que mensura a eficiência e a eficácia de uma ação. O sistema de avaliação de desempenho não pode ser tratado como único, pois há diferença entre culturas e entre as necessidades de diferentes organizações. Esses sistemas são formados por um conjunto de medidas de desempenhos individuais sofrendo influência do ambiente onde estão inseridas, conforme Figura 1.

Figura 1 - Dimensões da avaliação de desempenho

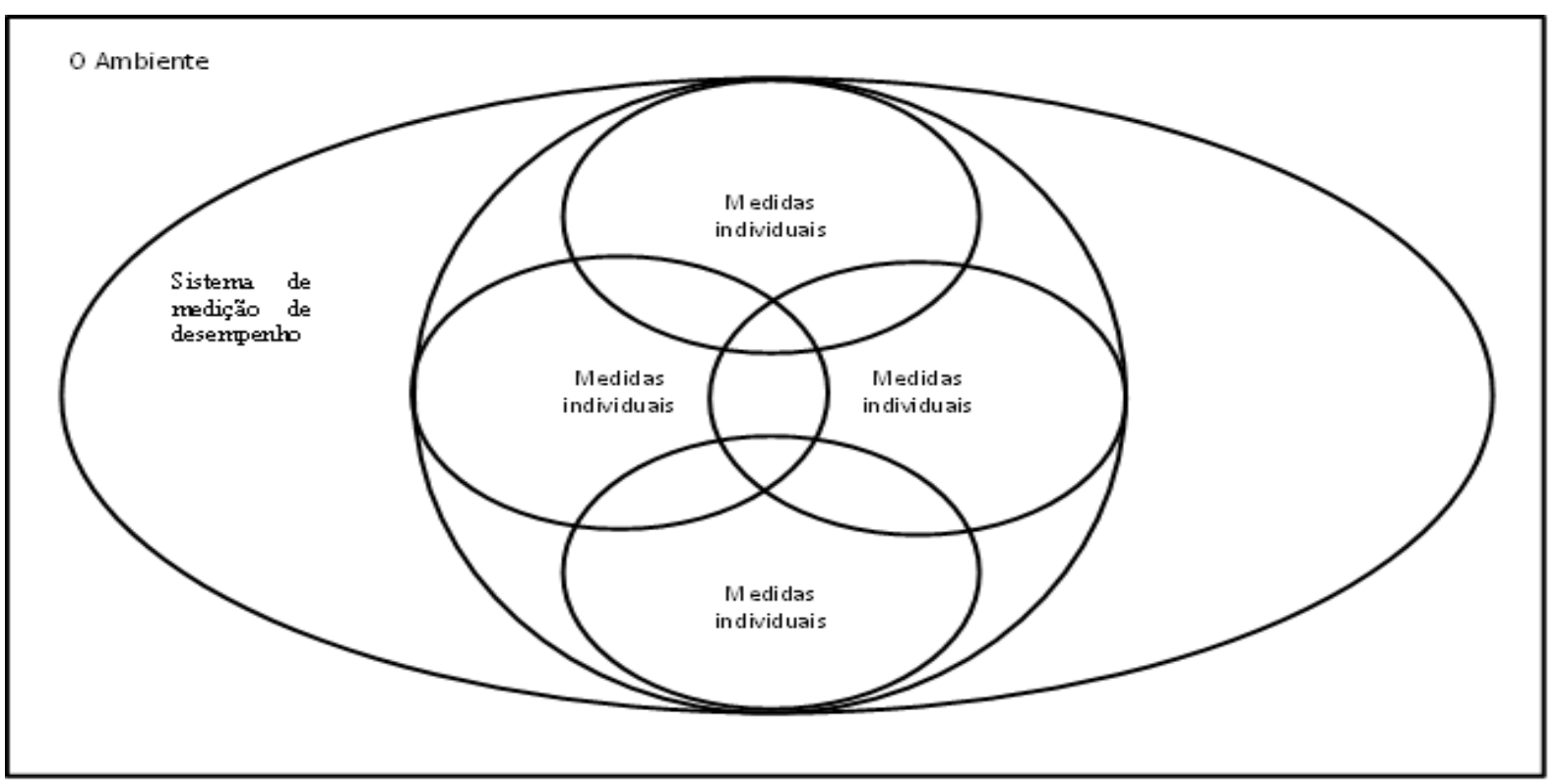

Fonte: Neely, Gregory e Platts (1995) 
A Avaliação de desempenho impacta inteiramente no ambiente em que opera. Decidir o que medir e como medir influencia indivíduos e grupos dentro da organização. Nessa mesma linha de pensamento Ferraz e Martins (2002, p.3) entendem que:

\begin{abstract}
[...] em termos de medidas de desempenho individuais, o diagnóstico avalia que medidas são usadas, para que elas são usadas, seu custo e que benefícios elas oferecem para a organização. No caso do SMD, o diagnóstico procura identificar se o sistema tem todos os elementos apropriados (interno, externo, financeiro e não-financeiro), se tem medidas de melhoria, se tem medidas que relatam objetivos de curto e de longo prazo, se suas medidas estão integradas verticalmente e horizontalmente e se há alguma medida de desempenho em conflito com outra.
\end{abstract}

O objetivo de um sistema de avaliação de desempenho é direcionar a organização para os melhores resultados. Silva (2010) coaduna com esse entendimento, pois entende que o sistema de avaliação de desempenho deve fornecer informações corretas, ajudando a organização a desenvolver ações de prevenção que a levem a atingir objetivos definidos. Já para Bourne et al. (2003), a avaliação de desempenho refere-se à utilização de um conjunto multidimensional de medidas de desempenho para o planejamento e a gestão de um negócio.

\title{
2.2 Avaliação de desempenho na área pública
}

A avaliação de desempenho não é aplicada somente em empresas privadas. As instituições públicas também necessitam utilizar desse mecanismo para melhor desenvolver suas atividades. $\mathrm{Na}$ área pública, a avaliação de desempenho ocorre da mesma forma que na área privada: surge da necessidade de instrumentos de controle para direcionar os gestores ao alcance de suas metas na organização. A avaliação de desempenho é também necessidade da sociedade enquanto usuária do sistema público, uma vez que ela paga tributos, na expectativa que o gestor aplique de forma correta os recursos e atenda aos anseios dela. O gestor público, tendo em vista fazer o uso correto dos recursos públicos, utilizará o processo de avaliação de desempenho para a tomada de decisões em sua gestão (MOREIRA; SANTOS; SILVEIRA, 2006).

O sistema de avaliação de desempenho, seja na área pública ou privada, tem por objetivo subsidiar os gestores no processo de tomada de decisão. Para Figueiredo et al.(2005), o sistema de avaliação de desempenho precisa fornecer informações aos gestores que possam auxiliá-los a perceber se a estratégia está sendo executada conforme o planejado e se continua sendo viável ao desenvolvimento da organização, na busca por melhor desempenho.

Segundo Evans e Bellamy (1995) a gestão pública tem sua avaliação de desempenho com base em controles e processos, ou seja, orçado versus despesas reais, ao invés de resultado e desempenho. Esta abordagem é ineficaz e força a gestão a concentrar-se no aspecto de responsabilidade no lugar de resultados.

\subsection{Tipologias de pesquisas}

Sobre o processo de pesquisa, Richardson (1999, p. 70) o caracteriza quanto ao método em quantitativa e qualitativa. Para o autor a pesquisa quantitativa é "o emprego da quantificação tanto nas modalidades de coleta de informações, quanto no tratamento delas por meio de técnicas estatísticas". No que se refere ao emprego da pesquisa qualitativa, o autor considera que essa metodologia pode "descrever a complexidade de determinado problema, analisar a interação de certas variáveis, compreender e classificar os processos dinâmicos vividos por grupos sociais” (1999, p. 80). No que concerne aos objetivos da pesquisa, Gil (2010) os classifica em descritivo, exploratório e explicativo. Collis e Hussey (2005) destacam a pesquisa descritiva como aquela que apresenta a conduta dos fenômenos, usada para identificar e obter elementos sobre as características de um determinado problema ou questão. Já a pesquisa exploratória, conforme Gil (2010), proporciona 
familiaridade com o problema, com o propósito de torná-lo explícito ou a construir hipóteses. Esse tipo de pesquisa, em geral, abrange levantamento bibliográfico e entrevistas com pessoas que tem conhecimento prático sobre o problema pesquisado. Sobre o emprego da pesquisa explicativa, o autor esclarece que ela visa identificar os fatores que causam ou colaboram para o acontecimento dos fatos. Aprofunda o conhecimento da realidade porque explica a razão, o "porquê" das coisas (GIL, 2010). Para a realização de uma pesquisa há vários procedimentos, tais como: pesquisa documental, bibliográfica, estudo de caso, Survey e pesquisa de campo. Segundo Marconi e Lakatos (2010), a pesquisa documental tem como característica sua fonte de coleta de dados, pois está limitado a documentos, escrito ou não, formando o que se chama de fontes primárias. Por outro lado, a pesquisa bibliográfica compreende toda a bibliografia já tornada pública em relação ao tema de estudo. Quanto ao estudo de caso, este nasce do anseio de compreender os fatos sociais complexos, esse procedimento permite que os investigadores detenham as características holísticas e significativas dos eventos da vida real (YIN, 2010). Já a survey, para Collis e Hussey (2005, p. 70), é onde "uma amostra de sujeitos é retirada de uma população e estudada para se fazerem inferências sobre essa população". No que concerne ao estudo de campo, Marconi e Lakatos (2010) afirmam que é a busca pelas informações ou conhecimentos a respeito de um problema, para a qual se busca uma resposta, ou de uma hipótese, que se queira comprovar, ou descobrir a relação e os fenômenos entre eles.

\subsection{Pesquisas anteriores}

Iniciativas de estudos semelhantes como este têm sido comum na literatura de gestão. Com base nas leituras realizadas sobre avaliação de desempenho, foram selecionados alguns estudos que apresentam relação com o tema para fundamentar a pesquisa.

O estudo apresentado por Keinert (2000), referente à administração pública. A autora analisou os artigos publicados entre 1937 e 1997 na Revista de Administração Pública (RAP) e na Revista do Serviço Público (RSP). Os resultados da pesquisa demonstraram que as discussões tecnicista e autoritária, devem ser substituídas por uma discussão mais ampla do papel do Estado.

Pacheco (2003) pesquisou sobre administração pública em revistas especializadas de 1995 a 2002. A autora concluiu que entre os estudos analisados, prevaleceu o estudo de caso como estratégia de pesquisa. A autora abalizou a tendência de pesquisadores citarem a si próprios, de diluição temática e de adoção acrítica de teorias desenvolvidas em outras disciplinas.

Lyrio, Ensslin e Dutra (2007) pesquisaram sobre avaliação de desempenho em organizações públicas, trazendo análise de uma amostragem de publicações acadêmicas nas áreas de administração e Contabilidade de 2002 a 2006. Os resultados apontaram que há uma diversidade de linhas de pensamentos referente à avaliação de desempenho em organizações públicas, verificando-se pela não reincidência de artigos publicados pelos mesmos autores e pela variedade de referências bibliográficas encontradas no estudo.

Hocayen-da-silva, Rossoni e Ferreira Junior (2008) estudaram sobre administração pública e gestão social: a produção científica brasileira entre 2000 e 2005. Os autores concluíram que a média de autores por artigo vem crescendo. A maioria dos estudos enquadrou-se como teórico-empírico, dos estudos empíricos a maioria utiliza o método qualitativo, com predomínio do estudo de caso como estratégia de pesquisa, no entanto o uso do método quantitativo vem aumentando.

Martins et al. (2010) realizaram um trabalho referente à avaliação de desempenho individual, no setor público brasileiro, onde analisou a produção acadêmica de 2000 a 2009. O estudo teve como resultado uma grande variedade de autores. $\mathrm{O}$ autor constatou que muitos dos autores dos trabalhos não pertenciam à área de estudo para compor o escopo teórico dos artigos, demonstrando certa dificuldade em estabelecer uma linha teórica sobre o assunto.

A identificação dos resultados alcançados por esses trabalhos pode trazer contribuições complementares ao presente estudo, tanto na construção do referencial teórico quanto na análise desta pesquisa. Outrossim, acredita-se que o tipo de análise proposto neste artigo também pode fornecer

R. Cont. Ufba, Salvador-Ba, v. 8, n. 2, p. 05 - 20, mai-ago 2014 
subsídios para dar continuidade a esses estudos, bem como favorecer no aumento da literatura para à área contábil e particularmente à avaliação de desempenho.

\section{METODOLOGIA DO ESTUDO}

Este trabalho buscou identificar e compilar os trabalhos associados ao tema de avaliação de desempenho no setor público, no âmbito dos trabalhos publicados em anais dos congressos qualificados pela Capes como "E1".

O Congresso de Controladoria e Contabilidade da USP tem contado com a participação de professores, pesquisadores, profissionais e alunos de todas as regiões do país, interessados na divulgação e avaliação dos seus estudos científicos. O evento tem se constituído em um espaço privilegiado para apresentação e discussão de conhecimentos na área (CCC-USP, 2012).

O Encontro da ANPAD - ENANPAD - realiza-se anualmente, no mês de setembro e é hoje o maior evento da comunidade científica e acadêmica de administração no país. É um grande incentivador da produção científica na área, sendo que, nos últimos três anos, em cada evento, cerca de 3000 trabalhos nas diversas áreas temáticas foram submetidos à apreciação, dos quais, aproximadamente, 800 foram apresentados (ANPAD, 2012).

A Associação Nacional de Programas de Pós-Graduação em Ciências Contábeis - ANPCONT tem a missão de ser a interlocutora dos Programas de Pós-Graduação em Ciências Contábeis perante os agentes com os quais se relaciona, contribuindo para o fortalecimento do ensino e a pesquisa nas suas várias áreas (ANPCONT, 2012).

Por fim, o Congresso Brasileiro de Custos (CBC) é o principal evento ligado à área de custos empresariais do Brasil, além disso, são os principais divulgadores da produção técnico-científica da especialidade e áreas afins, proporcionando a interação da comunidade acadêmica, pesquisadores, professores e estudantes, com empresários, consultores, contadores, administradores e demais profissionais atuantes na área da Gestão Estratégica de Custos (ABC, 2012).

A coleta de dados ocorreu por meio de pesquisa na plataforma dos sítios dos congressos mencionados. Foram separados os artigos que contivessem a expressão Avaliação de desempenho no setor público, no título, no resumo ou nas palavras-chave.

Sendo assim, as edições que não se encontravam disponíveis on-line para a pesquisa não foram consideradas. Aplicam-se nesse caso as edições de 2002 e 2003 do CCC-USP e a $18^{\text {a }}$ edição do CBC de 2011.

\section{APRESENTAÇÃo E ANÁLISE DOS RESULTADOS DA PESQUISA.}

4.1 Artigos referentes à avaliação de desempenho e avaliação de desempenho na área pública e suas características.

Seguindo os procedimentos descritos na metodologia, foram selecionados 117 artigos relativos à Avaliação de Desempenho. A maior quantidade de artigos está disponível nos congressos ENANPAD e CBC. Na Tabela 1 constam os dados de análise da pesquisa. Entre esses, também no ENANPAD encontram-se a maior parte dos artigos referentes ao setor público.

Tabela 1 - artigos referentes à avaliação de desempenho

\begin{tabular}{|l|c|c|}
\hline \multicolumn{1}{|c|}{ Congressos } & Avaliação de desempenho & Área pública \\
\hline ANPCONT & 0 & 0 \\
\hline CCC-USP & 11 & 1 \\
\hline CBC & 80 & 3 \\
\hline ENANPAD & 26 & 8 \\
\hline
\end{tabular}

R. Cont. Ufba, Salvador-Ba, v. 8, n. 2, p. 05 - 20, mai-ago 2014 


\begin{tabular}{|l|r|r}
\hline Total & 117 & 12 \\
\hline
\end{tabular}

Fonte: Elaborada pelos autores com base nos sites da ANPCONT, CCC-USP, CBC e ENANPAD.

Ao analisar a Tabela 1, verifica-se que dos 117 artigos encontrados, apenas 12 se referem ao assunto de interesse do estudo.

Observou-se que entre 2004 a 2011 houve apenas um artigo publicado sobre avaliação de desempenho, no CCC-USP, disponível na edição do $11^{\circ}$ Congresso (2011), intitulado "Divulgação de desempenho de órgão público: uma análise de conteúdo dos relatórios de gestão da entidade fiscalizadora superior brasileira". Neste artigo Castaldelli Junior e Aquino (2011) tiveram como objetivo analisar o uso de indicadores na divulgação de desempenho da entidade fiscalizadora superior brasileira, o Tribunal de Contas da União (TCU). Para alcançar o objetivo proposto, os autores utilizaram como procedimento metodológico a pesquisa quanti-qualitativa, estudo de caso e análise de documentos. A análise apontou que a divulgação de desempenho do TCU ainda tem como base grande parte em indicadores de saída, apesar da tendência em direção aos indicadores de resultados.

No CBC, foram encontrados três artigos que abordam avaliação de desempenho em órgãos públicos, no período de 2001 a 2010. Em 2006, no XIII CBC, foi apresentado o artigo "Análise crítica de métodos de avaliação de desempenho em instituições públicas de ensino superior" que realizou uma análise crítica de métodos de avaliação de desempenho em entidades públicas de ensino superior quanto ao cumprimento às determinações dos artigos 70 e 74 da Constituição Federal. A metodologia adotada foi a pesquisa qualitativa, exploratória, bibliográfica e documental. Os autores Moreira e Santos (2006) concluíram que os modelos de avaliação de desempenho analisados, não atendem integralmente às determinações dos artigos 70 e 74 da Constituição Federal.

Lyrio, Ensslin e Dutra (2007) apresentaram no XIV CBC o trabalho intitulado "Avaliação de desempenho em organizações públicas: análise de uma amostragem de publicações acadêmicas nas áreas de administração e Contabilidade de 2002 a 2006”. O objetivo deste estudo foi traçar o perfil da produção científica relacionada à avaliação de desempenho em organizações públicas, publicada nos anos de 2002 a 2006, em periódicos que abordam pesquisas das áreas de Administração e Contabilidade, classificados com conceito "A" no Sistema Qualis/Capes, triênio 2004/2006 e nos Anais do Encontro Nacional de Administração Pública e Governança (ENAPG) e do ENANPAD. Este estudo teve como metodologia a pesquisa quanti-qualitativa, exploratória e bibliográfica. Os resultados demonstraram que as pesquisas sobre a avaliação de desempenho em instituições públicas, ainda não apresentam números elevados, correspondendo a 3,87\% da amostra pesquisada da população de 671 artigos.

Ainda no XIV CBC, foi desenvolvido o estudo "Construção de um modelo de avaliação de desempenho da forma de distribuição da reserva de orçamento para contingências da universidade do estado de Santa Catarina" de autoria de Brotti, Ensslin e Ensslin (2007). Esse artigo teve por objetivo construir um modelo multicritério para avaliar o desempenho da forma de distribuição da Reserva de Orçamento para Contingências (ROC) da Fundação Universidade do Estado de Santa Catarina (UDESC). Para alcançar o objetivo proposto, o trabalho teve como procedimento metodológico a pesquisa quanti-qualitativa, exploratória e estudo de caso. $\mathrm{O}$ resultado da pesquisa apontou que o método utilizado como ferramenta de intervenção melhorou o entendimento sobre o problema estudado, bem como auxiliou positivamente na busca de novas perspectivas para a questão da Distribuição da Reserva de Orçamento para Contingências (DROC).

Ao pesquisar no ENANPAD, no período de 2002 a 2011, foram encontrados 8 artigos que tratam da avaliação de desempenho na área pública. Odelius e Santos (2006) pesquisaram sobre "Avaliação de desempenho individual no contexto da Administração Pública Federal direta: aspectos determinantes de sua efetividade". O artigo teve como objetivo identificar aspectos associados aos níveis organizacional, gerencial e individual de duas organizações da Administração Pública Federal direta (APFd) que, na percepção de avaliadores e avaliados, influenciam a efetividade dos sistemas de avaliação de desempenho. Para desenvolver a proposta de estudo, a metodologia adotada foi pesquisa, 
quanti-qualitativa e exploratória. Os resultados obtidos indicam que aspectos culturais e relativos ao preparo gerencial são os que mais influenciam a efetividade dos SAD e que a percepção sobre aspectos da avaliação é diferente, de acordo com características funcionais e demográficas dos servidores.

Narducci, Villardi e Dubeux (2006) apresentaram o trabalho "Uma proposta de análise quantitativa da avaliação de desempenho por competências para minimizar as restrições culturais do poder judiciário ao modelo de administração pública gerencial: o caso de um tribunal de justiça". O objetivo do artigo visou orientar a concepção e elaboração de uma metodologia de avaliação de desempenho por competências condizentes com a proposta de gestão por competências, implementada num Tribunal do Poder Judiciário. Para alcançar o objetivo proposto, a metodologia adotada foi a pesquisa qualitativa, exploratória e estudo de caso. Nesta Instituição despontaram-se limitações culturais ao modelo de administração pública gerencial, especificando estimas e descrições das equipes responsáveis pela implementação da avaliação de desempenho por competências, tais como: o "homem cordial" oposto ao "homem burocrático", que vive dialeticamente entre o "jeitinho" brasileiro e o formalismo.

O trabalho intitulado "Avaliação de desempenho de BPM na administração pública brasileira com o uso de análise multivariada" teve por objetivo adaptar o modelo teórico de avaliação de gerenciamento de processos de negócio BPM - disponível na revisão bibliográfica ao contexto dos processos de negócio para o Programa Nacional de Apoio aos Fiscos Estaduais (PNAFE). A metodologia adotada foi a pesquisa quantitativa, bibliográfica e survey. Os autores Sobreira Netto, Gouvêa e Ferreira (2006) chegaram à conclusão que as dimensões, análise crítica e aprendizado foram as que mais distinguiram o gerenciamento de processos classificados nas categorias satisfatórias e insatisfatórias.

O artigo "O processo de avaliação de desempenho individual no choque de gestão mineiro" teve por objetivo analisar o processo de avaliação de desempenho individual (ADI) adotado no governo mineiro, no âmbito da reforma da gestão pública denominada Choque de Gestão. Para alcançar o objetivo proposto Peci et al. (2008) trabalharam com a metodologia de pesquisa quantitativa e de campo. Os resultados revelaram as potencialidades do ADI e destacaram as peculiaridades da adoção do instrumento em organizações públicas, especificamente, a necessidade e a simultânea dificuldade de alinhar o ADI com os objetivos institucionais e com os objetivos maiores da reforma.

Marques, Albergaria e Lacerda (2008) pesquisaram "Inovações em gestão de pessoas no setor público: a visão dos servidores sobre a implantação da avaliação de desempenho individual pelo governo de Minas Gerais". O objetivo do artigo foi apresentar e discutir os resultados de uma pesquisa de campo que avaliou a percepção dos servidores públicos sobre as mudanças implementadas pelo Governo de Minas Gerais por intermédio do projeto Choque de Gestão, particularmente em relação à implantação da ADI. Para atingir o objetivo proposto, a metodologia adotada foi a pesquisa quantiqualitativa e estudo de caso. O resultado apontou que a maioria dos servidores concorda, coopera e divulga positivamente a ADI e não sofrem pressão de seus pares para resistir à implantação do processo de avaliação.

Em 2008, foi publicado o artigo "A avaliação de desempenho do servidor público brasileiro: uma análise da Lei n ${ }^{\circ} 14.694$ de 30/07/2003, do Estado de Minas Gerais". O objetivo deste estudo consistiu em fazer uma análise do tratamento que a avaliação de desempenho recebe (de forma genérica) no setor público brasileiro, como prática de gestão de pessoas. Na sequência é destacada a análise da legislação vigente no Estado de Minas Gerais que normatiza a avaliação de desempenho institucional dos servidores atuantes em seu Poder Executivo. A metodologia adotada constitui-se de pesquisa bibliográfica e documental. Nogueira (2008) concluiu que por questões culturais, há uma resistência à adoção de instrumentos gerenciais, descumprindo, assim, resoluções legislativas que determinam a prática de avaliação e acompanhamento do desempenho destes servidores públicos. Quanto à análise da Lei n 14.694/2003, de Minas Gerais, mostrou o valor norteador cultivado pela gestão do Estado 
em relação à avaliação de desempenho funcional, como um instrumento imparcial de gestão de pessoas.

Pereira (2009) apresentou o trabalho "Avaliação de desempenho na Polícia Militar do Espírito Santo" cujo objetivo foi identificar a presença de elementos da gestão burocrática e elementos da gestão empresarial, nas últimas décadas, na Polícia Militar do Espírito Santo e, em consequência, verificar como a organização vem se adaptando às novas demandas sociais. A metodologia adotada foi a pesquisa qualitativa, pesquisa descritiva e estudo de caso. Os autores concluíram que a prática da avaliação de desempenho é inexistente na Polícia Militar do Espírito Santo.

O trabalho "Gerenciamento de desempenho de servidores públicos: estudo sobre a cooperação e a resistência dos servidores à implantação da avaliação de desempenho individual pelo governo de Minas Gerais" é de autoria de Marques, Morais e Albergaria, apresentado em 2009. O estudo objetivou identificar o nível de cooperação e de resistência dos servidores públicos do estado de Minas Gerais em relação às mudanças implementadas pelo governo estadual nas políticas e nas práticas de gestão de pessoas a partir da implantação da ADI. O método adotado foi a pesquisa quantiqualitativa, descritiva, explicativa e estudo de caso. A conclusão evidenciou que os servidores ainda não estão convencidos de que a $\mathrm{ADI}$ e os demais instrumentos de gestão a ela associados serão capazes de extinguir as práticas do empreguismo e da influência política em prol de uma abordagem gerencialista para administrar pessoas no serviço público de Minas Gerais.

No Quadro 1 é apresentada a descrição geral (ano, edição do congresso, titulo e metodologia utilizada) dos doze artigos localizados.

Quadro 1 - Descrição das publicações sobre avaliação de desempenho na área pública.

\begin{tabular}{|c|c|c|c|c|}
\hline$\stackrel{2}{2}$ & 党 & 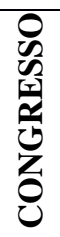 & TÍTULO & $\begin{array}{l}\text { MÉTODO DE PESQUISA } \\
\text { DECLARADO }\end{array}$ \\
\hline$\overline{\bar{\partial}}$ & $\stackrel{\sigma}{=}$ & 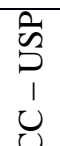 & $\begin{array}{l}\text { "Divulgação de desempenho de órgão público: uma } \\
\text { análise de conteúdo dos relatórios de gestão da } \\
\text { entidade fiscalizadora superior brasileira" }\end{array}$ & $\begin{array}{l}\text { Quanti-qualitativa, estudo de caso e } \\
\text { análise de documentos. }\end{array}$ \\
\hline ஓ & 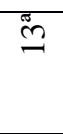 & U & $\begin{array}{l}\text { "Análise crítica de métodos de avaliação de } \\
\text { desempenho em instituições públicas de ensino } \\
\text { superior" }\end{array}$ & $\begin{array}{l}\text { Qualitativo, exploratório, } \\
\text { pesquisas bibliográfica e } \\
\text { documental. }\end{array}$ \\
\hline ঠ్ర & 导 & U & $\begin{array}{l}\text { "Avaliação de desempenho em organizações } \\
\text { públicas: análise de uma amostragem de publicações } \\
\text { acadêmicas nas áreas de administração e } \\
\text { Contabilidade de } 2002 \text { a } 2006 "\end{array}$ & $\begin{array}{l}\text { Quanti-qualitativa, exploratório e } \\
\text { pesquisa bibliográfica }\end{array}$ \\
\hline 尺े & $\stackrel{\sigma}{ \pm}$ & $\bigcup_{0}^{\infty}$ & $\begin{array}{l}\text { "Construção de um modelo de avaliação de } \\
\text { desempenho da forma de distribuição da reserva de } \\
\text { orçamento para contingências da universidade do } \\
\text { estado de Santa Catarina" }\end{array}$ & $\begin{array}{l}\text { Quanti-qualitativa, pesquisa } \\
\text { exploratória e } \\
\text { estudo de caso }\end{array}$ \\
\hline ஓ̊ & ¿্ল & 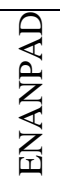 & $\begin{array}{l}\text { "Avaliação de Desempenho Individual no Contexto } \\
\text { da Administração Pública Federal direta: Aspectos } \\
\text { Determinantes de sua de Efetividade". }\end{array}$ & $\begin{array}{l}\text { Quanti-qualitativa e pesquisa } \\
\text { exploratória }\end{array}$ \\
\hline ஓ & :্লি & 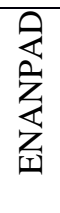 & $\begin{array}{l}\text { "Uma Proposta de Análise Quantitativa da Avaliação } \\
\text { de Desempenho por Competências para minimizar as } \\
\text { restrições culturais do Poder Judiciário ao Modelo de } \\
\text { Administração Pública Gerencial: O Caso de um } \\
\text { Tribunal de Justiça" }\end{array}$ & $\begin{array}{c}\text { Qualitativo, pesquisa exploratória e } \\
\text { estudo de caso }\end{array}$ \\
\hline
\end{tabular}




\begin{tabular}{|c|c|c|c|c|}
\hline ஓ̊ & ì & $\begin{array}{l}\text { 至 } \\
\text { Z } \\
\text { Z }\end{array}$ & $\begin{array}{l}\text { "Avaliação de Desempenho de } \text { BPM na } \\
\text { Administração Pública Brasileira com o Uso de } \\
\text { Análise Multivariada". }\end{array}$ & $\begin{array}{l}\text { Quantitativa, pesquisa bibliográfica, } \\
\text { e survey }\end{array}$ \\
\hline 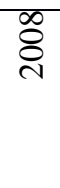 & $\stackrel{\circ}{\pi}$ & 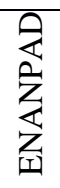 & $\begin{array}{l}\text { "O processo de avaliação de desempenho individual } \\
\text { no choque de gestão mineiro". }\end{array}$ & $\begin{array}{c}\text { Quantitativa e } \\
\text { pesquisa de campo. }\end{array}$ \\
\hline$\stackrel{\infty}{\bigodot}$ & $\stackrel{i}{n}$ & $\begin{array}{l}\text { 妾 } \\
\text { Z } \\
\text { Z }\end{array}$ & $\begin{array}{l}\text { "Inovações em Gestão de Pessoas no Setor Público: } \\
\text { a visão dos servidores sobre a implantação da } \\
\text { Avaliação de Desempenho Individual pelo Governo } \\
\text { de Minas Gerais". }\end{array}$ & Quanti-qualitativae estudo de caso \\
\hline$\stackrel{\infty}{\varnothing}$ & $\stackrel{\circ}{\pi}$ & $\begin{array}{l}\text { 竞 } \\
\text { Z } \\
\text { Z } \\
\text { Z }\end{array}$ & $\begin{array}{l}\text { "A Avaliação de Desempenho do Servidor Público } \\
\text { Brasileiro: uma análise da Lei no } 14.694 \text { de } \\
\text { 30/07/2003, do Estado de Minas Gerais". }\end{array}$ & $\begin{array}{l}\text { Pesquisas bibliográfica } \\
\text { e documental }\end{array}$ \\
\hline ஓे & $\dot{m}$ & 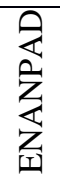 & $\begin{array}{l}\text { "Avaliação de desempenho na Polícia Militar do } \\
\text { Espírito Santo". }\end{array}$ & $\begin{array}{l}\text { Qualitativa, pesquisa descritiva e } \\
\text { estudo de caso. }\end{array}$ \\
\hline$\overline{\bar{\delta}}$ & in & $\begin{array}{l}\text { 玄 } \\
\sum_{Z}^{Z} \\
\text { Z }\end{array}$ & $\begin{array}{l}\text { "Gerenciamento de Desempenho de Servidores } \\
\text { Públicos: Estudo sobre a Cooperação e a Resistência } \\
\text { dos servidores à Implantação da Avaliação de } \\
\text { Desempenho Individual pelo Governo de Minas } \\
\text { Gerais" }\end{array}$ & $\begin{array}{c}\text { Quanti-qualitativa, } \\
\text { pesquisas descritiva e explicativa, e } \\
\text { estudo de caso. }\end{array}$ \\
\hline
\end{tabular}

Fonte: Elaborado pelos autores com base nos sites da ANPCONT, CCC-USP, CBC e ENANPAD.

No Quadro 1, os artigos relacionados ao tema, de forma geral, mostram um enfoque empírico e teórico-empírico. O procedimento de pesquisa que mais foi evidenciado pelos pesquisadores foi o de pesquisa exploratória, pesquisa bibliográfica e o estudo de caso. Os resultados dessa análise corroboram com o trabalho de Lyrio, Ensslin e Dutra (2007), Pacheco (2003) e Hocayen-da-silva, Rossoni e Ferreira Junior (2008) que a maioria dos estudos enquadrou-se como teórico-empírico, dos estudos empíricos, a maioria utiliza o estudo de caso como estratégia de pesquisa.

\section{2 - Artigos por segmentos da área pública}

Na Tabela 2 é apresentada a distribuição dos artigos por segmento da área pública, sendo dividida em ensino, segurança, gestão de pessoas e outros.

Tabela 2: classificação dos artigos por segmento da área púbica

\begin{tabular}{|l|c|c|c|c|c|}
\hline $\begin{array}{l}\text { Segmento da área } \\
\text { pública }\end{array}$ & ANPCONT & $\begin{array}{c}\text { Congresso } \\
\text { USP }\end{array}$ & CBC & ENANPAD & Total \\
\hline Ensino & 0 & 0 & 2 & 0 & 2 \\
\hline Segurança & 0 & 0 & 0 & 1 & 1 \\
\hline Gestão de Pessoas & 0 & 0 & 0 & 6 & $\mathbf{6}$ \\
\hline Outros & 0 & 1 & 1 & 1 & 3 \\
\hline Total & $\mathbf{0}$ & $\mathbf{1}$ & $\mathbf{3}$ & $\mathbf{8}$ & $\mathbf{1 2}$ \\
\hline
\end{tabular}

Fonte: Elaborado pelos autores com base nos sites da ANPCONT, CCC-USP, CBC e ENANPAD. 
Evidencia-se pela análise da Tabela 2 que há maior interesse dos pesquisadores em desenvolver trabalhos referentes à avaliação de desempenho no segmento de gestão de pessoas, constatando um total de 6 artigos. Nesse sentido, percebe-se que os segmentos menos citados, como os temas que envolvem ensino e segurança, não despertam o interesse dos estudiosos da área pública.

\subsection{Número de autores}

Na Tabela 3 são apresentados os artigos classificados por número de autores. Observa-se que os artigos são publicados, em sua maioria, por mais de um autor, havendo equilíbrio entre os artigos publicados, constando de dois a três autores. Os resultados dessa análise correspondem aos do trabalho de Cardoso, Pereira e Guerreiro (2004), Hocayen-da-silva, Rossoni e Ferreira Junior (2008) e Lyrio, Ensslin e Dutra (2007) que ponderavam que a forma mais utilizada de parceria corresponde a trabalhos apresentados por mais de um autor.

Tabela 3: número de autores por artigo

\begin{tabular}{|c|c|c|c|c|c|}
\hline $\begin{array}{c}\text { Número de } \\
\text { autores }\end{array}$ & ANPCONT & Congresso USP & CBC & ENANPAD & Total \\
\hline 1 & & & & 2 & 2 \\
\hline 2 & & 1 & 1 & 2 & 4 \\
\hline 3 & & & 2 & 3 & 5 \\
\hline Mais de 3 & & & & 1 & 1 \\
\hline Total & $\mathbf{0}$ & $\mathbf{1}$ & $\mathbf{3}$ & $\mathbf{8}$ & $\mathbf{1 2}$ \\
\hline
\end{tabular}

Fonte: Elaborado pelos autores com base nos sites da ANPCONT, CCC-USP, CBC e ENANPAD.

Ao analisar o número de publicações sobre o tema, por autor, fica constatado que não existe uma continuidade nas pesquisas com este enfoque, uma vez que dos 31 autores entre os 12 artigos encontrados sobre a avaliação de desempenho na área pública, apenas dois autores têm mais de um artigo publicado, conforme constatado na Tabela 4.

Tabela 4: autores com mais de um artigo

\begin{tabular}{|l|c|c|c|c|c|c|}
\hline Autores & $\begin{array}{l}\text { Universidade de } \\
\text { origem dos autores }\end{array}$ & ANPCONT & CCC-USP & CBC & ENANPAD & Total \\
\hline $\begin{array}{l}\text { Antônio Luiz } \\
\text { Marques }\end{array}$ & UFMG & 0 & 0 & 0 & 2 & 2 \\
\hline $\begin{array}{l}\text { Ariane Rocha } \\
\text { Albergaria }\end{array}$ & FEAD & 0 & 0 & 0 & 2 & 2 \\
\hline
\end{tabular}

Fonte: Elaborado pelos autores com base nos sites da ANPCONT, CCC-USP, CBC e ENANPAD.

Na Tabela 5 são relacionados os autores que mais aparecem nas referências dos artigos dos congressos em análise. Sendo considerados aqueles que foram citados mais de três vezes.

Tabela 5 - autores mais referenciados

\begin{tabular}{|l|c|}
\hline \multicolumn{1}{|c|}{ Nome dos autores } & Frequência \\
\hline ENSSLIN, Sandra Rolim. & 5 \\
\hline BOYNE, G.A. & 4 \\
\hline MARINI, Caio. & 4 \\
\hline MOTTA, F. C. Prestes. & 4 \\
\hline NEELY, A. D. & 4 \\
\hline VILHENA, Renata. & 4 \\
\hline
\end{tabular}

Fonte: Elaborado pelos autores com base na ANPCONT, CCC-USP, CBC e ENANPAD.

Do total de 453 autores referenciados, identifica-se que a autora mais referenciada é Sandra Rolim Ensslin, que desenvolve pesquisas voltadas para a área pública. Nesta etapa da pesquisa, a intenção é evidenciar o autor com mais influência nos trabalhos que se referem à área publica. Porém, percebese que os autores dos artigos analisados não dão a continuidade no tema abordado nos trabalhos. Isso 
pode significar que ainda há a necessidade de exploração sobre o assunto, com a finalidade de se aumentar a literatura existente na área pública sobre avaliação de desempenho.

\subsection{Número de obras referenciadas}

É apresentado na Tabela 6 um recorte das obras mais referenciadas nos artigos encontrados na pesquisa, destacando aquelas que aparecem mais de 2 vezes.

Tabela 6 - obra mais citada

\begin{tabular}{|c|c|}
\hline OBRAS & REFERÊNCIAS \\
\hline $\begin{array}{l}\text { VILHENA, Renata; MARTINS, Humberto Falcão.; MARINI, Caio.; GUIMARÂES, } \\
\text { Tadeu Barreto. O choque de gestão em Minas Gerais - políticas da gestão pública para o } \\
\text { desenvolvimento. Belo Horizonte: Editora UFMG, } 2006 \text {. }\end{array}$ & 4 \\
\hline $\begin{array}{l}\text { HAIR, Joseph F.; ANDERSON, Rolph E.; BACK, William C. Multivariate Data Analysis. } \\
4 \text { ed. New Jersey Prentice Hall, Inc. } 1995 .\end{array}$ & 3 \\
\hline
\end{tabular}

Fonte: Elaborado pelos autores com base na ANPCONT, CCC-USP, CBC e ENANPAD.

Dentre 280 obras referenciadas, destaca-se a obra "O choque de gestão em Minas Gerais - políticas da gestão pública para o desenvolvimento". Também consta na Tabela 6, outra obra muito referenciada, entretanto não está relacionada com o tema de estudo. Isso demonstra uma diversidade de linhas de pensamentos, o que confirma o entendimento de Lyrio, Ensslin e Dutra (2007) que pesquisaram sobre avaliação de desempenho, análise da amostragem das publicações acadêmicas e Martins et al. (2010) que investigaram a avaliação de desempenho individual no setor público brasileiro, análise das produções acadêmicas.

\section{CONCLUSÃO}

A caracterização da natureza e utilidade da avaliação de desempenho na área pública, como uma prática que viabilize maior eficiência e eficácia na execução das atividades de apoio, é um tema pouco presente nos congressos em análise, considerando os números apresentados na seção 4. Esta conclusão se dá com base na identificação de apenas doze artigos apresentados nas edições de Congressos Brasileiros "E1" da área contábil, que abordaram a temática avaliação de desempenho na área pública.

Constatou-se que os artigos publicados nos eventos selecionados têm predominância de evidência quanti-qualitativa. Ademais, foi possível identificar que o estudo de caso e a pesquisa bibliográfica são uns dos procedimentos técnicos com maior presença na amostra.

Pela análise depreendida pode-se verificar que os artigos publicados concentram-se mais na área de gestão de pessoas e, geralmente, são publicados por mais de um autor.

Os resultados apontaram que a autora mais influente é Sandra Rolim Ensslin. A obra mais referenciada é a obra "o choque de gestão em Minas Gerais - políticas da gestão pública para o desenvolvimento" de organização de VILHENA, Renata; MARTINS, Humberto Falcão; MARINI, Caio; GUIMARÂES, Tadeu Barreto.

Dentre os dados observados, compreende-se que os estudos de avaliação de desempenho na área pública não têm uma continuidade, salvo o caso de dois autores que tiveram duas publicações a respeito do assunto.

O número de obras referenciadas também enfatiza tal afirmação, uma vez que de um total considerado, 280 obras, apenas duas são citadas mais de três vezes. Dentre essas, apenas uma se refere ao tema, demonstrando que os estudos realizados pelos autores parecem não estar concentrados numa área específica.

Considerando os objetivos deste artigo, os resultados auferidos e as conclusões apresentadas, sugerese a realização de estudos que possam discutir os motivos que levam ao baixo índice de artigos 
relativos ao tema. Também se considera importante realizar pesquisas que procuram analisar as causas que levam os autores não darem continuidade nas pesquisas envolvendo o mesmo. Recomenda-se também estudos semelhantes a este, baseando-se em periódicos e congressos nacionais e internacionais.

Espera-se que os resultados deste estudo, retratando a acentuada falta de pesquisas nessa temática de avaliação de desempenho na área pública, motivem os pesquisadores brasileiros a explorarem as alternativas de pesquisa que aqui se descortinam.

\section{REFERÊNCIAS}

ABC - Associação Brasileira de Custos. Objetivos. Disponível em: <http://www.abcustos.org.br/congresso/view?ID_CONGRESSO=22>. Acesso em: 12 mar. 2012.

ANPCONT - Associação Nacional de Programas de Pós-Graduação em Ciências Contábeis. Matéria. Disponível em: <http://www.anpcont.com.br/site/materia.php?id=2>. Acesso em: 12 mar. 2012.

BOURNE, Mike; NEELY, Andy; MILLS, John. PLATTS, Ken. Implementing performance measurement systems: a literature review. Int. J. Business Performance Management, Vol. 5, 1, 2003.

BROTTI, Vera; ENSSLIN, Sandra Rolim; ENSSLIN, Leonardo. Construção de um modelo de avaliação de desempenho da forma de distribuição da reserva de orçamento para contingência da universidade do estado de Santa Catarina. In: CONGRESSO BRASILEIRO DE CUSTOS, XIV., 2007, João Pessoa - PB. Anais... João Pessoa: ABC, 2007.

CARDOSO, R. L; PEREIRA, C. A; GUERREIRO, R. A Produção Acadêmica em Custos no Âmbito do ENANPAD: uma Análise de 1998 a 2003. In: Encontro Nacional de Programas de PósGraduação em Administração, XXVIII, 2004, Curitiba. Anais... Curitiba: ENANPAD 2004.

CASTALDELLI JUNIOR, Eduardo; AQUINO, André Carlos Busanelli. Análise de conteúdo dos relatórios de gestão da entidade fiscalizadora superior brasileira. In: CONGRESSO USP DE CONTROLADORIA E CONTABILIDADE, $11^{\circ}$.; 2011, São Paulo - SP. Anais... São Paulo: CCC - USP, 2011.

CARPINETTI, L. C. R. Uma proposta para o processo de identificação e desdobramento de melhorias de manufatura: uma abordagem estratégica. Tese Livre Docência. Escola de Engenharia de São Carlos, Universidade de São Paulo, São Carlos, 2000.

COLLIS, Jill, HUSSEY, Roger. Pesquisa em administração: um guia prático para alunos de graduação e pós-graduação. 2 ed. Porto Alegre: Bookman, 2005.

DIEHL, Carlos Alberto, SOUZA, Marcos Antonio de. Publicações Sobre o Custeio Baseado em Atividades (ABC) em Congresso Brasileiros de Custos no período de 1997 a 2006. Revista Contabilidade Vista e Revista, Minas Gerais, v. 19 n. 4, p 39 - 57, 2008.

ENANPAD - Encontro da Anpad. Eventos. Disponível em:

<http://www.anpad.org.br/eventos.php>. Acesso em: 12 mar. 2012. 
EVANS, Patricia; BELLAMY, Sheila. Performance evaluation in the Australian public sector the role of management and cost accounting systems. International Journal of Public Sector Management, v. 8, n.6, 1995, p $30-58,1995$.

FERRAZ, Claudia Augusto, MARTINS, Roberto Antonio. Um método abrangente para o diagnostico da medição de desempenho. In: ENCONTRO NACIONAL DE ENGENHARIA DE PRODUÇÃO, XXII., 2002, Curitiba - Paraná. Anais... Curitiba - Paraná: ENEGEP, 2002.

FIGUEIREDO, Moacyr Amaral Domingues. SOARES, Teresia Diana Lewe Van Aduard de Macedo. FUKS, Saul. FIGUEIREDO, Lívia Cavalcanti. Definição de atributos desejáveis para auxiliar a auto - avaliação dos novos sistemas de medição de desempenho organizacional. Gestão \& Produção, São Carlos, v.12, n.2, p.305-315, 2005.

GHALAYINI, Alaa M.; NOBLE, James S.The changing basis of performance measurement. International Journal of Operation \& Production Management.V. 16, n. 8, p. 63 - 80, 1996.

GIL, Antonio Carlos. Como elaborar projetos de pesquisa. 5 edição. São Paulo: Atlas 2010.

HOCAYEN-DA-SILVA, Antônio João, ROSSONI, Luciano, FERREIRA JUNIOR, Israel. Administração pública e gestão social: a produção cientifica brasileira entre 2000 e 2005 . Revista de Administração Pública, v. 42, n. 4, p. 655-680, 2008.

KEINERT, T. M. M. O que é administração pública no Brasil? In: ENCONTRO DA ASSOCIAÇÃO NACIONAL DE PROGRAMAS DE PÓS-GRADUAÇÃO EM ADMINISTRAÇÃO, 24., 2000, Florianópolis. Anais... Florianópolis: Anpad, 2000.

KIYAN, Fabio Makita. Proposta para desenvolvimento de indicadores de desempenho como suporte estratégico. Dissertação (Mestrado) - Escola de Engenharia de São Carlos, Universidade de São Paulo, São Carlos, 2001.

LIMA, Andréa Cavalcante Correia. PONTE, Vera Maria Rodrigues. Um estudo sobre os fatores chaves na implantação de modelos de medição de desempenho organizacional. Revista de Administração e Contabilidade da Unisinos, São Leopoldo, v.3, n. 3, p. 285 - 296, 2006.

LYRIO, Maurício Vasconcellos Leão; ENSSLIN, Leonardo; DUTRA, Ademar. Avaliação de desempenho em organizações públicas: análise de uma amostragem de publicações acadêmicas nas áreas de administração e Contabilidade de 2002 a 2006. In: CONGRESSO BRASILEIRO DE CUSTO, XIV., 2007, João Pessoa - PB. Anais... João Pessoa: CBC, 2007.

MARCONI, Marina de Andrade; LAKATOS, Eva Maria. Fundamentos de metodologia cientifica. 7. ed. São Paulo: Atlas, 2010.

MARQUES, Antônio Luiz; ALBERGARIA, Ariane Rocha; LACERDA, Mariana Soares Lacerda. Inovações em gestão de pessoas no setor público: a visão dos servidores sobre a implantação da avaliação de desempenho individual pelo governo de Minas Gerais. In: ENCONTRO DA ASSOCIAÇÃO NACIONAL DE PÓS - GRADUAÇÃO E PESQUISA EM ADMINISTRAÇÃO, 32 ${ }^{\circ}$, 2008, Rio de Janeiro- RJ. Anais... Rio de Janeiro - Rio de Janeiro: ENANPAD 2008.

MARQUES, Antônio Luiz; MORAIS, Kelly de; ALBERGARIA, Ariane Rocha. Gerenciamento de desempenho de servidores públicos: estudo sobre a cooperação e a resistência dos servidores à 
implantação da avaliação de desempenho individual pelo governo de Minas Gerais. In: ENCONTRO DA ASSOCIAÇÃO NACIONAL DE PÓS - GRADUAÇÃO E PESQUISA EM ADMINISTRAÇÃO, 35², 2011, Rio de Janeiro-RJ. Anais... Rio de Janeiro: ENANPAD 2011.

MARTINS, BibianaVolkmer; MARQUES, Juliana Rodrigues; VIEIRA, Luciano José Martins; GARAY, Angela Beatriz Scheffer. Avaliação de desempenho individual no setor público brasileiro: análise da produção acadêmica de 2000 a 2009. In: ENCONTRO DE ADMINISTRAÇÃO PÚBLICA E GOVERNANÇA, Vitória - ES. Anais... Vitória: EnAPG 2010.

MOREIRA, Ney Paulo; SANTOS, Nálbia de Araújo; SILVEIRA. Análise crítica da aplicação de métodos de avaliação de desempenho em uma instituição de ensino superior. In: CONGRESSO BRASILEIRO DE CUSTOS, XIII., 2006, Belo Horizonte - BH. Anais... Belo Horizonte: CBC, 2006.

NARDUCCI, Viviane.; VILLARDI, Beatriz Quiroz.; DUBEUX, Veranise. Uma proposta de análise quantitativa da avaliação de desempenho por competências para minimizar as restrições culturais do poder judiciário ao modelo de administração pública gerencial: o caso de um tribunal de justiça. In: In: ENCONTRO DA ASSOCIAÇÃO NACIONAL DE PÓS - GRADUAÇÃO E PESQUISA EM ADMINISTRAÇÃO, 30, 2006, Salvador - Bahia. Anais... Salvador - Bahia: ENANPAD 2006.

NEELY, A., MILLS, J., GREGORY, M., PLATTS, K., Performance measurement system design - a literature review and research agenda, International Journal of Operations and Production Management, p. 80-116, 1995.

NOGUEIRA, José Marcelo. A avaliação de desempenho do servidor público brasileiro: uma análise da lei no 14.694 de 30/07/2003, do Estado de Minas Gerais. In: ENCONTRO DA ASSOCIAÇÃO NACIONAL DE PÓS - GRADUAÇÃO E PESQUISA EM ADMINISTRAÇÃO, 32º, 2008, Rio de Janeiro- RJ. Anais... Rio de Janeiro - Rio de Janeiro: ENANPAD, 2008.

ODELIUS, Catarina Cecília; SANTOS, Paulo Ricardo Godoy. Avaliação de desempenho individual no contexto da administração pública federal direta: aspectos determinantes de sua efetividade. In: ENCONTRO DA ASSOCIAÇÃO NACIONAL DE PÓS - GRADUAÇÃO E PESQUISA EM ADMINISTRAÇÃO, 30, 2006, Salvador - Bahia. Anais... Salvador - Bahia: ENANPAD, 2006. PACHECO, Regina Silvia. Administração pública nas revistas especializadas - Brasil, 1995- 2002. Revista de Administração de Empresas, São Paulo, v. 43, n. 4, p. 63-71, out./dez. 2003.

PECI, Alketa; LEMOS, Carolina Siqueira; MENESES, Cecilia Milagros Rosas; FARIA, Daniela de Melo; VÉRAS, Marcelo Lima. O processo de avaliação de desempenho individual no choque de gestão mineiro. In: ENCONTRO DA ASSOCIAÇÃO NACIONAL DE PÓS - GRADUAÇÃO E PESQUISA EM ADMINISTRAÇÃO, 32², 2008, Rio de Janeiro- RJ. Anais... Rio de Janeiro - Rio de Janeiro: ENANPAD, 2008.

PEREIRA, José Dirceu. Avaliação de desempenho na Polícia Militar do Espirito Santo. In: ENCONTRO DA ASSOCIAÇÃ̃O NACIONAL DE PÓS - GRADUAÇÃO E PESQUISA EM ADMINISTRAÇÃO, 33º, 2009, São Paulo- SP. Anais... São Paulo - São Paulo: ENANPAD, 2009.

RICHARDSON, Roberto Jarry. Pesquisa Social: métodos e técnicas. 3. ed. São Paulo: Atlas, 1999.

SECCHI, Leonardo. Modelos organizacionais e reformas da administração pública. Revista de Administração Pública. Rio de Janeiro, v. 43, n. 2, p. 347-369, 2009. 
SILVA, Wilson Ricardo Cardoso. Métricas para avaliação de desempenho organizacional baseada em indicadores de qualidade. Revista Fibra e Ciência, n. 03, v. 2, 2010.

SOBREIRA NETTO, Francisco; GOUVÊA, Maria Aparecida; FERREIRA, João Eduardo. Avaliação de desempenho de BMP na administração pública brasileira com o uso de análise multivariada. In: ENCONTRO DA ASSOCIAÇÃO NACIONAL DE PÓS - GRADUAÇÃO E PESQUISA EM ADMINISTRAÇÃO, 30, 2006, Salvador - Bahia. Anais... Salvador - Bahia: ENANPAD, 2006.

TEZZA, Rafael; BORNIA, Antonio Cezar; VEY, Ivan Henrique. Sistema de medição de desempenho: uma revisão e classificação da literatura. Gestão de Produção, São Carlos, v.17, n1, p. $75-93,2010$.

USP - CONGRESSO CONTROLADORIA E CONTABILIDADE. Disponível em:

<http://www.congressousp.fipecafi.org/sobre-congresso.asp>. Acesso em: 12 mar. 2012.

YIN, Robert K. Estudo de caso: planejamento e método. Porto Alegre: Bookmam. 4. ed. 2010. 